\title{
Increases in paradoxical and slow sleep during the acquisition of an appetitive task in rats
}

\author{
CARLYLE SMITH, DOUG LOWE, and MARY JANE SMITH \\ Trent University, Peterborough, Ontario, Canada K9J 7B8
}

\begin{abstract}
Rats were trained to barpress for food reward. In a preliminary experiment, a $171 / 2-\mathrm{h}$, daily, partial selective paradoxical sleep (PS) deprivation was alternated with a $6 \frac{1}{2} 2$-h rest period. Training was given just before the rest period each day, and EEG and EMG were continuously recorded during the rest periods. PS was observed to increase during task acquisition. These increases were of a prolonged nature, lasting the entire rest period. Extinction training did not appear to alter PS levels. In a more detailed, 24-h/day, continuous recording experiment, the same task was given. No sleep deprivation was imposed, and the animals were normally rested. Both PS and slow-wave sleep (SWS) were found to be increased during the training sessions. Possible relationships between these sleep parameter changes and learning are discussed.
\end{abstract}

Two main paradigms have recently been used in attempts to demonstrate a relationship between paradoxical sleep (PS) and some type of learning or memory consolidation mechanism in animals. The first type has investigated the effects of PS deprivation on learning performance. Several studies have reported some type of learning retardation as a result of the deprivation treatment (Fishbein, 1970, 1971; Leconte \& Bloch, 1970; Leconte \& Hennevin, 1973; Leconte, Hennevin, \& Bloch, 1974; Pearlman, 1971, 1973; Pearlman \& Becker, 1973, 1974a, 1974b; Pearlman \& Greenburg, 1973; Stern, 1971).

The second paradigm has involved the measurement of changes in sleep parameters during and after task acquisition. Several of these studies have reported increased PS during the first few hours of sleep following the training sessions (Leconte \& Hennevin, 1971; Lucero, 1970). In other work (Leconte et al., 1973), it has been reported that these PS increases were most prominent in the first $1 / 2 \mathrm{~h}$ of sleep following the training session and were due to an increased number of PS periods, provided the number of trials per session was kept short. In yet another study, PS increases have been reported following a barpress acquisition, extinction, and reacquisition (Hennevin, Leconte, \& Bloch, 1974). In that experiment, however, the PS increases were evident during the full $3 \mathrm{~h}$ of recording time.

Unfortunately, all of the above-mentioned studies have a recording time not exceeding $3 \mathrm{~h}$. This procedure severely limits the amount of information on sleep changes accompanying learning. In continuous sleep recording studies using two strains of mice in a 5-day complex shock avoidance discrimination task, two types of PS increase have been observed (Smith, Kitahama, Valatx, \& Jouvet, 1972, 1974). A "shortterm" increase in PS appeared as the learning per- formance reached asymptotic levels, and this increase seemed to be due to an increased number of PS periods. This increase was confined to the first $1 / 2 \mathrm{~h}$ of sleep after sleep onset following the daily training session. A prolonged, or "'long-term,"' increase in PS appeared in an earlier phase of the learning experience, being manifest in the $24-48$-h period prior to a maximum increase in correct maze performance. This prolonged increase in PS was due neither to the number of PS periods nor to an increase in PS average size alone (Smith et al., 1974). Prolonged increases in PS following a shock avoidance acquisition in mice has also been reported by other workers which apparently were of a long-term nature (Fishbein, Kastaniotis, \& Chattman, 1974). By contrast, no short-term PS increases were observed in relation to task acquisition in this study, although marked changes in SWS were also reported.

Thus, PS and possibly even slow-wave sleep (SWS) appear to be related to task acquisition, but the data are incomplete and conflicting. The bulk of the studies reporting short-term PS increases have not explored beyond a 3 -h recording period (Hennevin et al., 1974; Leconte \& Hennevin, 1971; Leconte et al., 1974; Lucero, 1970). Only two studies have recorded long enough to report the presence of a long-term type of PS increase (Fishbein et al., 1974; Smith et al., 1974).

\section{EXPERIMENT 1}

The present studies were done in an attempt to determine if both the short-term and long-term PS increases would be manifest during a simple appetitive barpress acquisition when sleep recording was extended beyond $3 \mathrm{~h}$. 


\section{Method}

Subjects and Procedure. Subjects were 11 male rats of the Walker-Walker strain raised at the Trent University animal facility, weighing $300-450 \mathrm{~g}$ at the time of operation. All animals were surgically implanted with cortical (EEG) and electromyographic (EMG) electrodes. Each EEG electrode consisted of a smalldiameter wire soldered to a jeweller's screw. Four of these electrodes were then screwed into the skull bone. EMG electrodes were four small-diameter wires implanted in the neck and temporal muscles. All electrode leads were secured and affixed to a plug by means of dental acrylic such that the plug could be connected to a recording cable. At any given time during the experiment, EEG was recorded from two of the four EEG leads while EMG was recorded from two of the four EMG electrode leads. This gave one EEG and one EMG trace on the polygraph. After 12-14 days of recovery from the operation, the animals were continuously acclimatized to the recording cables for 6-8 days. Following this habituation period, the animals were selectively deprived of PS for $42 \mathrm{~h}$ by means of the "swimming pool" technique. Inverted flower pots, with a base diameter of $8.5 \mathrm{~cm}$, were placed in individual plastic pails. These served as islands for each animal when the pails were filled with water to the level of the base of the pots.

Following this initial continuous deprivation, a partial PS deprivation schedule was begun. Each morning at $1030 \mathrm{~h}$, the animals were removed from their pots and placed in individual glasssided recording cages and attached to the recording cable. Wet mash was freely available during this $61 / 2-\mathrm{h}$ rest period. The rats usually cleaned and ate for the first $1 / 2 \mathrm{~h}$ of the rest period and then slept a great deal for the next $6 \mathrm{~h}$. At $1700 \mathrm{~h}$ each day, the animals were again placed in the PS deprivation situation until $1030 \mathrm{~h}$ the following morning. This regime, as has been found in previous work (Smith \& Miskiman, 1975), results in subjects exhibiting at least $30 \%$ PS during the rest period, with only a slight tendency to reduced PS levels as days continue. This technique provides a high concentration of PS during the rest period and is economical in terms of EEG chart paper and experimenter time.

The laboratory was continuously illuminated during the course of this experiment, and, although the animals had been previously accustomed to a day-night cycle, the severity of this deprivation situation was believed to be enough to override the cyclic component.

The learning task. The task was a simple Skinner box barpress response with continuous reward schedule. All test animals $(\mathbf{N}=$ 6) were given a magazine (M) training day in the Skinner box for $45 \mathrm{~min}$ prior to the rest period. On this day, all subjects were trained to associate the sound of the "click" with a pellet in the food cup. No attempt was made to associate a barpress with presentation of food reward.

On the first training day (TD1) (day following M), the test animals were shaped to press the bar for food reward by utilizing the method of successive approximation. The animals were considered to have learned when 10 unaided responses were made within a 5-min period. There was also a second (TD2) and third (TD3) training day, followed by 2 days of extinction training (E1 and E2). On these latter 2 days, the rats were placed in the Skinner boxes for $45 \mathrm{~min}$ and food reward was withheld for barpressing. Barpress responses were automatically recorded on chart recorders.

The control animals $(\mathrm{N}=5)$ were given an equivalent $45 \mathrm{~min}$ per day in the Skinner boxes. They were allowed to eat a number of food pellets approximately equal to those eaten by the test animals. However, no training of any kind was given. All animals were closely watched during the 45 -min periods in the Skinner box to ensure that they were awake at all times.

\section{Results}

The learning task. Animals in the test group unintentionally fell into two distinct categories, with
Table 1

Individual Barpress Scores

\begin{tabular}{cccccc}
\hline \multirow{2}{*}{$\begin{array}{c}\text { Animal } \\
\text { Number }\end{array}$} & \multicolumn{5}{c}{ Training Day } \\
\cline { 2 - 6 } & TD1 & TD2 & TD3 & E1 & E2 \\
\hline 1 & 20 & 88 & 156 & 146 & 54 \\
2 & 46 & 275 & 249 & 90 & 20 \\
3 & 39 & 133 & 114 & 64 & 45 \\
4 & 0 & 113 & 149 & 42 & 9 \\
5 & 0 & 68 & 160 & 74 & 44 \\
6 & 0 & 10 & 251 & 140 & 78 \\
Mean & 17.5 & 114.5 & 179.8 & 92.7 & 41.7 \\
\hline
\end{tabular}

Note-The fast-learning (FL) animals, 1, 2, and 3, learned on TD1. The slow-learning (SL) animals, 4, 5, and 6, did not learn to barpress until TD2. The numbers indicate the total number of barpress responses made by each animal on each day.

three rats reaching criterion on TD1 and three rats on TD2. The number of barpresses for each animal per daily session can be seen in Table 1 .

The sleep phenomena. The electroencephalographic (EEG) and electromyographic (EMG) records allowed the categorization of the behavior into "awake," SWS, and PS. Low-voltage, fast-wave EEG activity coupled with pronounced EMG activity was classified as "awake." High-voltage, slow-wave EEG coupled with reduced EMG activity was considered to be SWS. Low-voltage, fast EEG with an almost complete absence of EMG activity was scored as PS. Extracted data included PS in minutes, SWS in minutes, and number of PS periods (number of times that PS occurred). The PS average size was calculated by dividing the number of minutes of PS by the number of PS periods.

In analyzing these measures to compare test and control groups, each animal was compared not only with control animal values but with his own mean baseline level as well. Since each animal was initially subjected to 2 consecutive days of baseline sleep recording, these data were averaged to provide a mean baseline value for each test and control animal. Difference scores were obtained by subtracting the mean baseline value of each animal from each of the training and extinction day values in both test and control groups. Test and control animal difference scores were compared in overall analyses of variance, using the unweighted means solution for unequal numbers of subjects per group. Individual orthogonal comparisons between test and control group values on $\mathrm{M}, \mathrm{TD} 1$, TD2, TD3, and E1 were then performed (Winer, 1971).

Considering first the PS measure, there was no overall difference between groups, nor was there any Groups by Trials interaction, although there was a significant trials effect $(F=3.60, \mathrm{df}=5 / 45$, $\mathrm{p}<.01)$ due to the overall variability from day to day in both groups. From the individual comparisons, a significant difference between test and control animals occurred on TD1 $(F=11.59, \mathrm{df}=1 / 9$, 


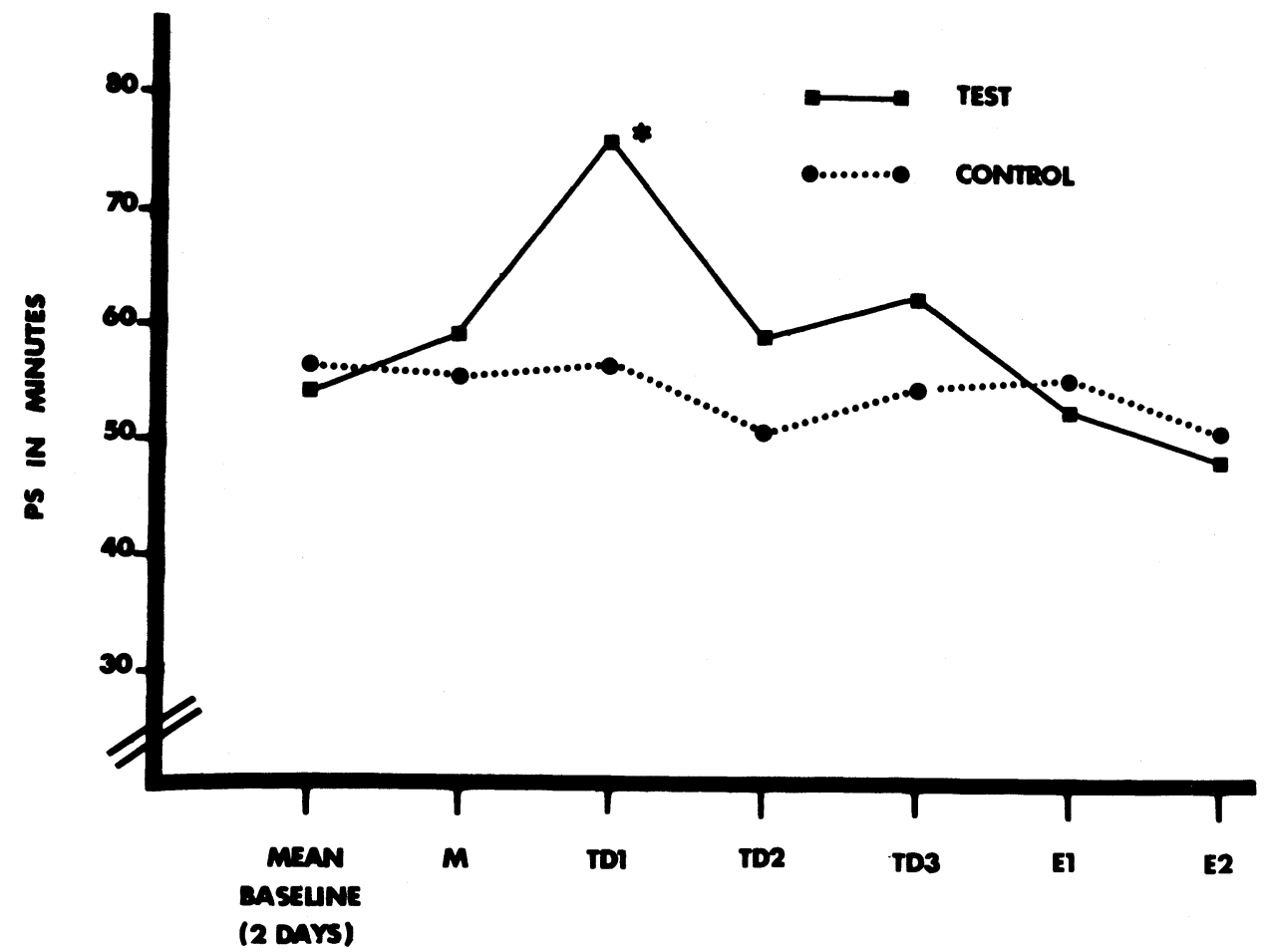

Figure 1. The mean number of minutes of PS observed during each daily rest period for test and control animals $\left({ }^{\star} p<.01\right)$.

$\mathrm{p}<.01)$. Although there appeared to be a slight elevation of PS for the test group on TD2 and TD3 as well, these differences were not statistically reliable. No difference was found between groups on E1.

Figure 1 shows the PS levels, in minutes, of test and control groups for each day of the experiment. The overall analysis of the number of PS periods revealed only a significant trials effect $(F=3.44$, $\mathrm{df}=5 / 45, \mathrm{p}<.01$ ), again due to the day-to-day fluctuations for both test and control groups. None of the orthogonal comparisons were significant. A similar overall analysis of variance and orthogonal comparisons for the SWS data revealed no significant differences.

Because the test animals clearly fit into two groups in terms of their learning performance, post hoc analyses were performed, comparing the control animals that had learned on TD1. In terms of PS, the mean increase for these three fast learning (FL) animals over their own mean baseline levels was $77 \%$, while for the three slow learning (SL) animals, which failed to reach criterion until TD2, the mean PS increase was only $15 \%$. The overall analysis of variance for PS comparing FL and control animals showed only a significant trials effect $(F=3.76$, $\mathrm{df}=5 / 30, \mathrm{p}<.01$ ). From the individual orthogonal comparisons, the test group value was greater than that of the controls $(F=11.25, \mathrm{df}=1 / 6, \mathrm{p}<.05)$. Similar analyses of the SL group with controls showed no significant differences present. The two subgroups are plotted separately with control values in Figure 2. Analyses of SWS, number of PS periods, and PS average size for the FL group revealed no differences at any point compared to controls.

Because of the "short-term" PS increase observed in previous experiments (Smith et al., 1974), an overall analysis of variance including $\mathbf{M}$ and the training and extinction days was performed. PS for the first $1 / 2 \mathrm{~h}$ after sleep onset following the daily session was used. Neither the overall analysis nor the individual comparisons of TD1, TD2, and TD3 revealed any significant differences.

\section{EXPERIMENT 2}

Despite the encouraging results of this experiment in terms of PS increases observed, it was felt that the selective PS deprivation regime might distort the actual nature of the PS increases, both in time of occurrence and in magnitude. Consequently, it was decided to do a second experiment employing continuous EEG recording learning of the same appetitive task.

\section{Method}

Eight animals were operated on as in Experiment 1 and habituated to the recording cables. Three days $(72 \mathrm{~h})$ of baseline recording was carried out, the beginning of the session being $1600 \mathrm{~h}$. A daynight cycle was imposed on these animals and the dark part of the 


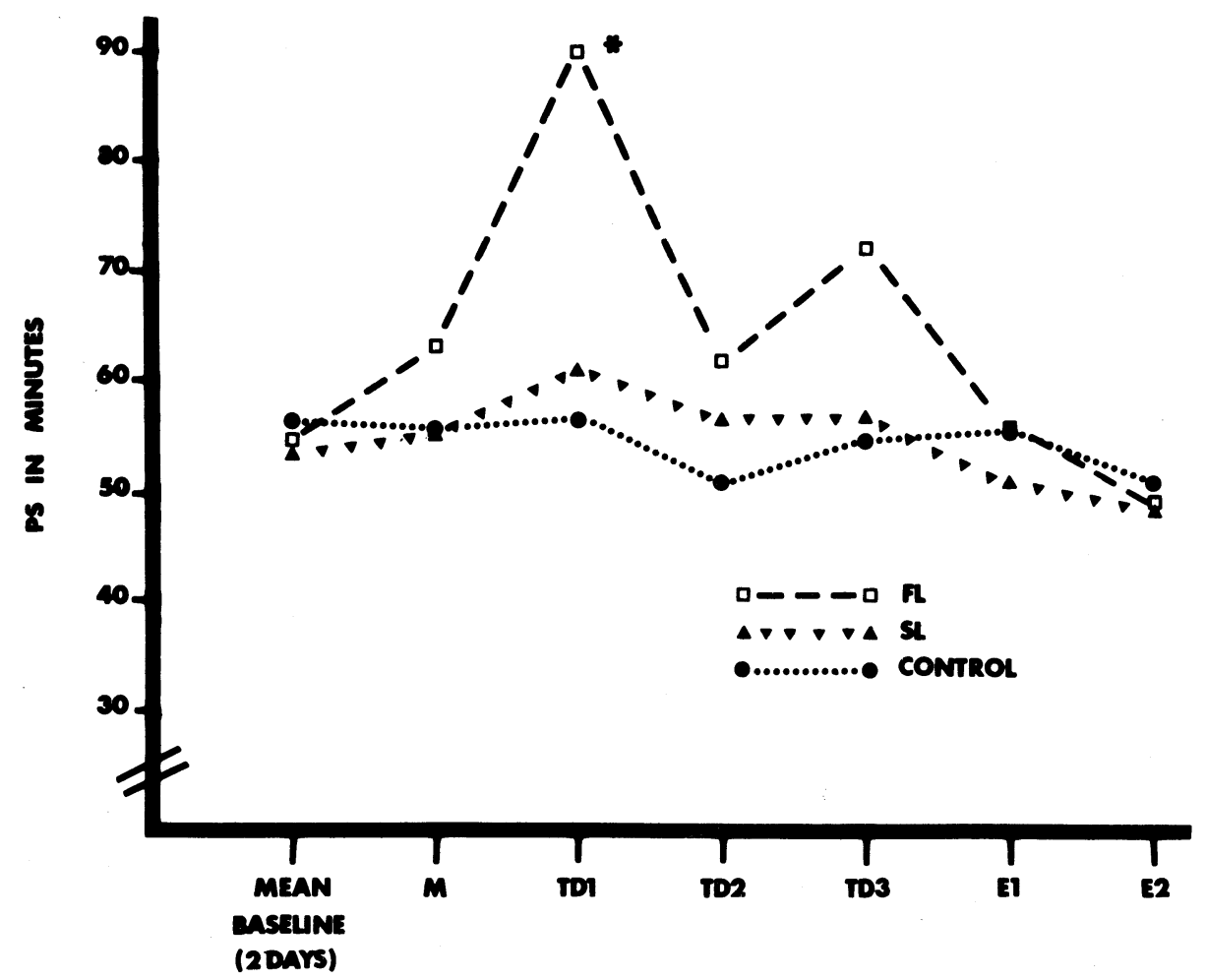

Figure 2. The mean number of minutes of PS observed during each daily rest period for FL, SL, and control group animals $\left({ }^{*} p<.05\right)$.

cycle began at $2000 \mathrm{~h}$, while the light part of the cycle began at $0800 \mathrm{~h}$. All animals were placed on a 1-h/day feeding schedule when the cable habituation was begun. The daily feeding of wet mash occurred between 1600 and $1700 \mathrm{~h}$ each day. After the 3 days of baseline sleep recording, four test animals were placed in separate Skinner boxes for $45 \mathrm{~min}$ prior to the feeding session. These animals were not fed and no "clicks" were presented. This was "box" control (BC) day and was intended to check the possibility of changes in sleep parameters resulting simply from a novel environment. The following day, magazine training was given as described previously in Experiment 1 . This was followed on succeeding days by three training sessions (TD1-TD3). One 24-h period of EEG recording was carried out on the day following TD3. No extinction training was given in this study as Experiment 1 had indicated that it did not appear to affect sleep parameters. Control animals $(\mathrm{N}=4)$ lived in their home cages for the entire experiment, being fed with test animals at the same time each day. Care was taken to ensure that the control animals remained awake during the $45 \mathrm{~min}$ that the test animals were being trained.

\section{Results}

The learning task. All four test animals learned to press the bar for food reward on TD1. Thus TD2 and TD3 resulted only in an increased number of barpresses during the 45-min daily test period.

The sleep phenomena. As in Experiment 1, the values of all sleep measures used in the test vs. control comparisons consisted of difference scores. Scores for a particular day consisted of the difference between the mean baseline value for each animal and the score of that same animal on a particular test day. Mean baseline values in this experiment consisted of the mean of the values for the first 3 days $(72 \mathrm{~h})$ of recording. As in Experiment 1, all measures were treated in the same way. First, an overall analysis of variance comparing the test with control group scores was performed, then individual orthogonal comparisons comparing test with control group values on each of BC, M, TD1, TD2, and TD3.

Considering first the PS measure (minutes/24-h period), the overall analysis of variance revealed no significant group differences or interaction. There was a significant trials difference $(F=5.17, \mathrm{df}=$ $5 / 30, p<.005$ ) due to the day-to-day fluctuations of both test and control groups. The only significant individual orthogonal comparison was on TD3, with the PS value of the test group being larger than that of the control group $(\mathrm{F}=17.45, \mathrm{df}=1 / 6, \mathrm{p}<.01)$. The PS average size measure was not significant in the overall analysis, but on TD1 the test group had a higher value for this measure than the control group $(F=7.25, \mathrm{df}=1 / 6, p<.05)$. For the number of PS periods measure, only the trials factor was significant in the overall analysis $(F=5.02, \mathrm{df}=$ $5 / 30, p<.005)$. However, none of the individual comparisons were significantly different.

For the SWS measure, the overall analysis of variance resulted in a significant difference between groups $(F=11.42, \mathrm{df}=1 / 6, \mathrm{p}<.025)$ and a trials 
effect $(F=5.04$, df $=5 / 30, p<.005)$ due to the day-to-day fluctuations in both groups.

Individual comparisons revealed significant differences at $\mathrm{BC}(\mathrm{F}=7.76, \mathrm{df}=1 / 6, \mathrm{p}<.05)$, at $\mathrm{M}$ $(\mathrm{F}=21.83, \mathrm{df}=1 / 6, \mathrm{p}<.005)$, at TD1 $(\mathrm{F}=13.67$, $\mathrm{df}=1 / 6, \mathrm{p}<.025)$, and at TD3 $(\mathrm{F}=43.15$, df $=$ $1 / 6, p<.001)$. All of these sleep parameters can be seen in Figure 3.

In looking at the measures related to PS (PS, number of PS periods, PS average size), it seemed clear that TD1 and TD3 were the most important days to consider in this experiment. First, the PS value on TD3 was larger for the test group than for the control group, while on TD1, the PS average size measure was larger for the test group. Further, it was only on these 2 days that PS for the test group was above mean baseline levels. For these reasons, detailed analyses of these two 24 -h periods were carried out, blocking the data into 4-h periods for both test and control groups. Individual analyses of variance of all the measures at 4-h intervals on the 2 test days revealed the following information: For TD1, PS was
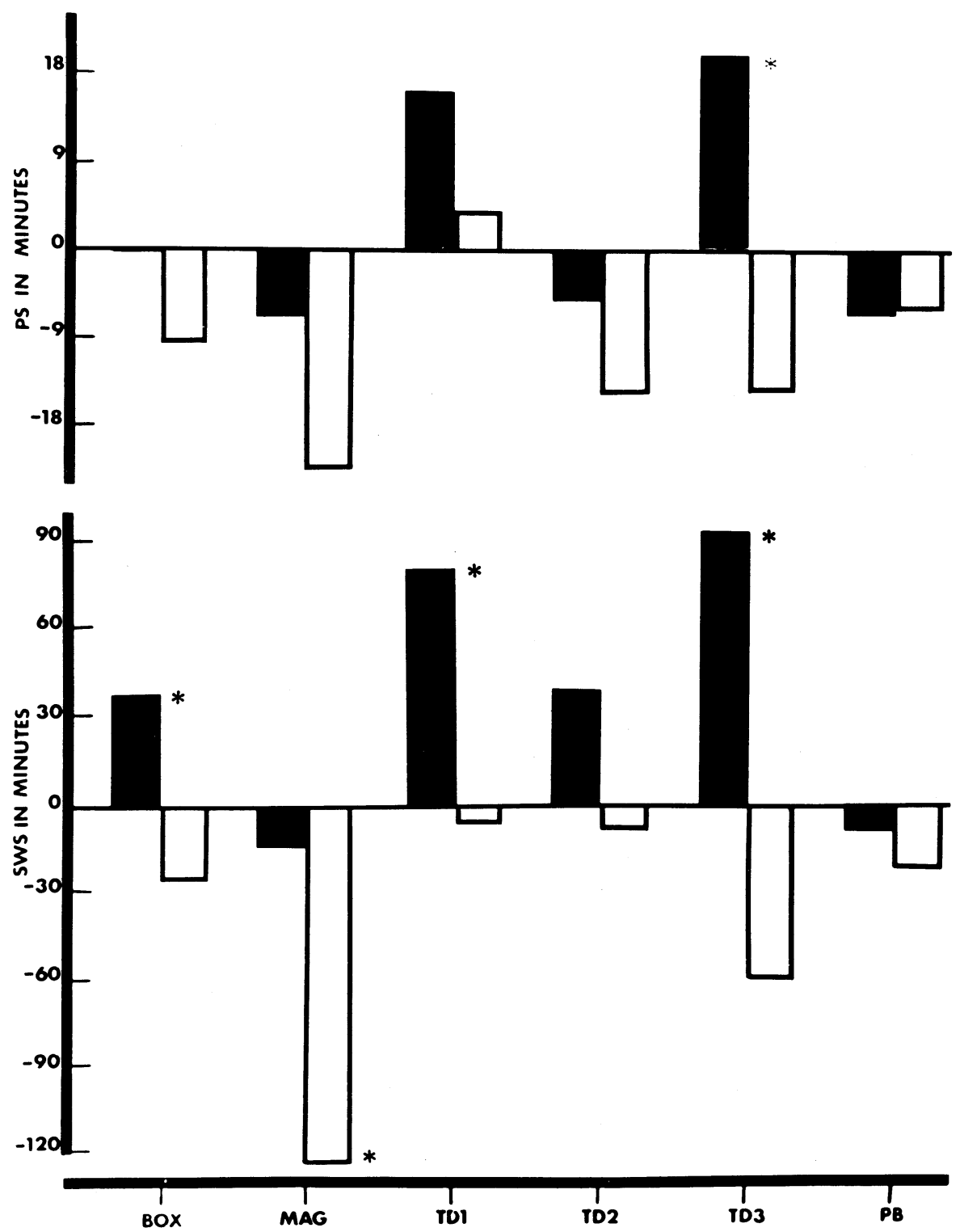

Figure 3. Bars represent the mean number of minutes of PS or SWS for each group above or below mean baseline levels. Solid bars are test group values and open bars are control group values. For the PS graph, ${ }^{*}=\mathbf{p}<.01$. For the SWS graph on BOX (BC), ${ }^{*}$ p $<.05$; on MAG (M), $*=p<.005$; on TD1, * $=p<.025$, and at TD3, * $=p<.001$. PB is the posttraining 24-h period of baseline recording. 
larger in test than in control group animals during the 2000-2359 time period $(F=7.30, \mathrm{df}=1 / 6$, $\mathrm{p}<.05)$. This increase appeared to be due to a significant increase in PS average size which was present during the same time period $(F=19.05, \mathrm{df}=1 / 6$, $\mathrm{p}<.005)$. On TD3, the PS measure was again elevated during the 2000-2359 time period $(F=10.64$, $\mathrm{df}=1 / 6, \mathrm{p}<.05)$, this time apparently due to a larger number of PS periods, which was significant during this same period of time $(F=9.04, \mathrm{df}=$ $1 / 6, p<.05)$. The same general pattern was observed in the SWS measure, with the test group SWS mean value being significant on TD1 during the 2000-2359 time period $(\mathrm{F}=8.32, \mathrm{df}=1 / 6, \mathrm{p}<.05)$ and the 2400-0359 time period $(F=148.28$, df $=1 / 6$, $\mathrm{p}<.001)$. On TD3, the SWS measure was elevated for a total period of $12 \mathrm{~h}$ in the test group, being significantly larger during the $2000-2359(F=6.90$, $\mathrm{df}=1 / 6, \mathrm{p}<.05), 2400-0359(\mathrm{~F}=6.40, \mathrm{df}=1 / 6$, $\mathrm{p}<.05), 2400-0359(\mathrm{~F}=9.34, \mathrm{df}=1 / 6, \mathrm{p}<.05)$, and 0400-0759 ( $\mathrm{F}=12.52, \mathrm{df}=1 / 6, \mathrm{p}<.05)$ time periods. Figure 4 shows the sleep measures at $4-h$ intervals on TD3.

In order to check once again for the short-term effect, an overall analysis of variance of the PS observed during the first $1 / 2 h$ of sleep following the daily session was performed. This was followed by individual comparisons on TD1, TD2, and TD3. No significant differences of any kind were observed.

\section{DISCUSSION}

Two experiments indicate that PS does increase during acquisition of an appetitive task. In fact, these increases are more complex and prolonged than expected. Further, from Experiment 2, it seems clear
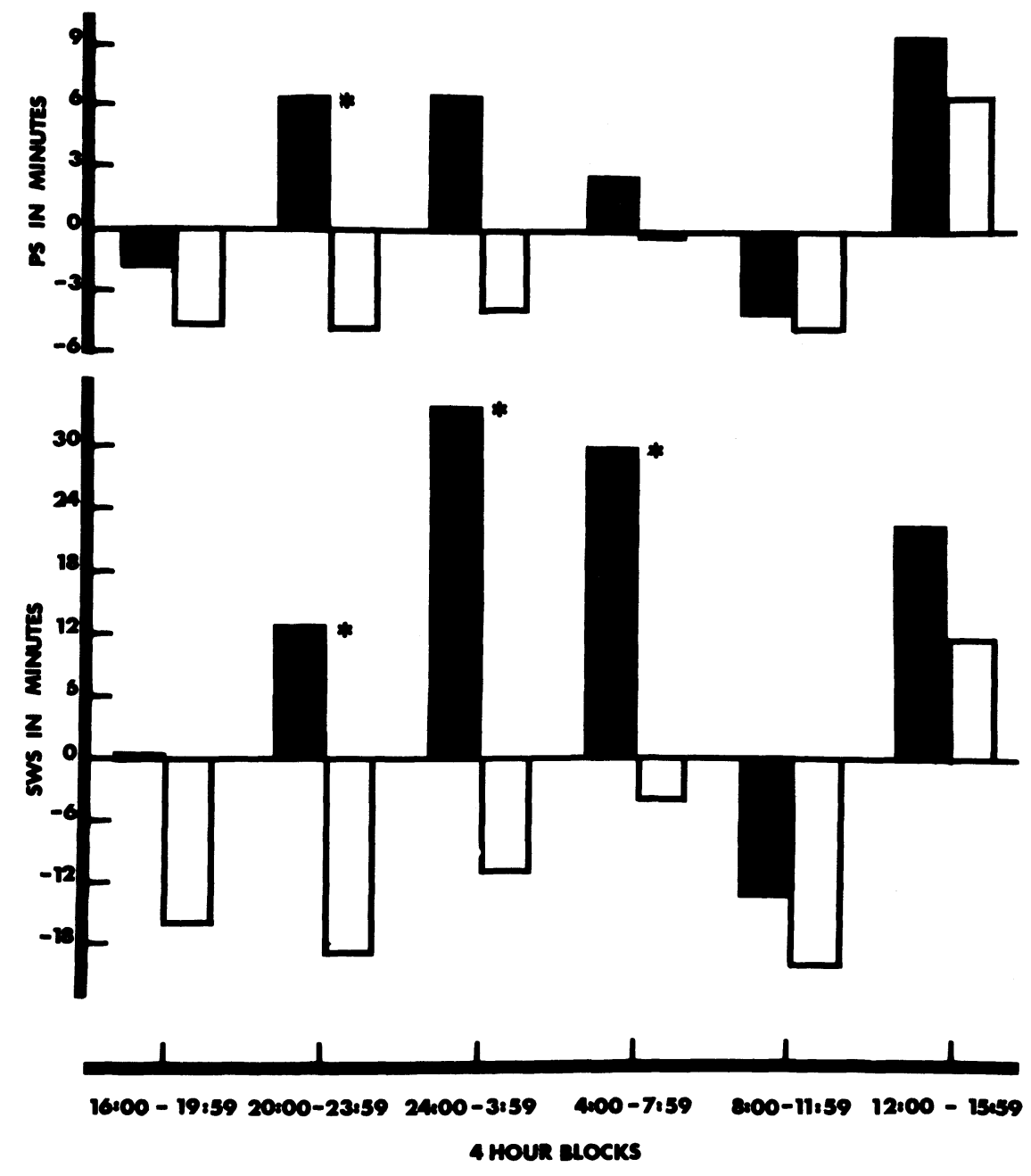

Figure 4. Bars represent the mean number of minutes of PS or SWS for each group above or below the mean baseline value for each 4-h block on TD3. Solid bars are test group values and open bars are control group values. For the PS graph, ${ }^{*}=p<.05$. For the SWS graph from 2000-2359, * $=p<.05$; from 2400-0359, * $=p<.05$; from 0400-0759, * $=p<.05$. 
that SWS also increases during task acquisition as well as on M day.

Considering the PS changes, the apparent differences in exactly when the maximum PS increases occur are somewhat obscured by the mean values. In Experiment 1, the largest PS increases appear to occur on TD1, while in Experiment 2, they appear to occur on TD3. In Experiment 1, the three FL animals had increases on TD1. Two of these animals showed a maximum PS on TD1, with smaller increases on TD3, but the third showed the PS maximum on TD3, although there was also a sizable PS increase on TD1. In Experiment 2, all four of the animals were in the FL category. Three of the four had the maximum PS increase on TD3, while one animal had the maximum on TD1. Thus, all animals in both experiments in the FL groups had two PS "peaks," one on TD1 and one on TD3. Attempts to correlate behavior more closely with PS maximums were not successful. Possibly this in part is due to the nature of the task, since it does not lend itself to exact numbers of trials in a given test session.

It might be argued that the PS increases on TD3 (Experiment 2) reflect motor activity rather than a learning process. That is, acquisition was apparently complete on TD1. Sessions on TD2 and TD3 simply gave the animals the opportunity for increasing the number of barpresses in the 45-min period. This motor interpretation is unlikely for many reasons. First, from Experiment 1, considerable motor activity was apparent on E1. The number of presses on E1 actually exceeded those on TD1 for every animal. Yet no changes in PS were observed following E1. In another learning task, a discrimination avoidance task, there was considerable motor activity, although the task was very simple. Test animals were compared with a control group that was not allowed this motor activity. No differences in PS were found (Smith \& Lowe, 1975). In previous work with other species (Smith et al., 1974), PS increases were observed during acquisition of a complex Y-maze shock avoidance task. One of the control groups included animals that were forced to run a much longer distance in each session because the light cues to the safe arms of the maze were removed. Yet, this group was no different from control animals that had no opportunity for motor behavior at all.

In both Experiments 1 and 2, PS average size and number of PS periods contributed to the PS increases observed. In Experiment 1, both are slightly, but not significantly, elevated. In Experiment 2, PS average size is significantly elevated on TD1, while the number of PS periods is only slightly elevated. On TD3, neither of these measures is significantly elevated. It seems very unlikely that either measure alone completely accounts for the PS increases on either day, and in terms of earlier results, the PS increases observed appear to have the general characteristics of the long-term, or prolonged, PS increases reported previously (Smith et al., 1974). Somewhat unlike the previously mentioned long-term effect was the fact that the present PS increases in Experiment 2 were so pronounced at particular time periods (2000-2359 on TD1 and 2000-0359 on TD3). Previous results in mice (Smith et al., 1974) showed small, nonsignificant, increases in PS throughout a 24-48-h period which in total were larger than the PS values of controls. Possible reasons for these differences include the longer training sessions in the present experiment $(45 \mathrm{~min}$ vs. $15 \mathrm{~min}$ ). Further, acquisition was achieved in one session in the present work, and these sleep changes were observed following acquisition, while in the previous experiments (Smith et al., 1974), this longterm effect was evident prior to major changes in learning performance. Prolonged PS increases which began several hours after the training session have also been reported by other workers (Fishbein et al., 1974). One other unique result from Experiment 2 was the increase in SWS. The pattern of these increases on TD1 and TD3 was essentially the same in character as the PS increases, with the exception that they persisted for a longer time ( $8 \mathrm{~h}$ on TD1, $12 \mathrm{~h}$ on TD3). More difficult to explain are the differences on BC and M control days. The test group increases in SWS on BC day might be considered to reflect some environmental or cognitive adjustment. However, this could not be the entire explanation of the SWS increases in this experiment, as they are most dramatic following TD1 and TD3, the training sessions. Further, they begin at exactly the same time as the PS increases. The possibility of "visual overload" might also be considered a contributing factor to the SWS increases. This idea (Horne, 1976) relates increases in SWS to large amounts of visual activity. However, since the length of time that the rats were in the training situation was not large (45 min/day), this hypothesis seems less likely to be an adequate explanation of the SWS increases observed. The levels of SWS for M day show both test and control group values below baseline levels. The extra low levels of SWS in the control group appear to be mainly due to one animal. The reason for that low value is not clear. Thus, although cognitive adjustment and visual overload may contribute somewhat to the levels of SWS observed, it would seem most likely that SWS was, to a large extent, involved in the learning task. SWS was presumably not evident in Experiment 1 because of the nature of the PS deprivation situation. The inverted flower pots do not prevent SWS, and, although no animals were recorded during the PS deprivation period, it is likely that the animals were able to get SWS (Dement et al., 1967).

Other workers have not found SWS to be uniquely 
involved with the learning process. SWS elevations have been reported in noncontingent shock control animals that received a large number of shocks (Leconte \& Hennevin, 1971). Fishbein et al. (1974) have also reported that shock plays a factor in SWS increases. Such increases seemed to be related to the shock situation and were observed in both experimental shock avoidance animals and in the yoked controls. It is interesting to note that the present work and those of quoted studies which report any SWS changes all had training sessions in excess of 45 min (Fishbein et al., 1974; Leconte \& Hennevin, 1971). Comparable studies with daily sessions of 20 min or less do not report SWS changes (Hennevin et al., 1974; Leconte et al., 1973; Smith et al., 1974).

A phenomenon not found in these studies is the short-term PS increases of Smith et al. (1974). Others (Leconte et al., 1973) have also previously reported this short-duration (about $1 / 2 \mathrm{~h}$ ) PS increase which appears shortly after sleep onset following the actual training session. Its prominence is greatest as learning reaches asymptotic levels and is due to an increased number of PS periods (Leconte et al., 1973). Although it is not clear why this phenomenon is missing, it would appear at the moment that the length of training session is again important. As well as the present results, data from two other studies provide support for this idea (Fishbein et al., 1974; Leconte \& Hennevin, 1971). The training sessions of these studies were all longer than $45 \mathrm{~min}$. Studies showing this short-term effect all had training sessions of $20 \mathrm{~min}$ or less.

One of the clearest results from Experiment 1 was the difference in PS between the FL and SL animals. Unfortunately, we cannot assume that somehow these larger amounts of PS for the FL group are due to a greater amount of learned material. Such an idea is too simple to incorporate the results of other laboratories. Leconte et al. (1973) have reported results very similar to those of the present studies, but Fishbein et al. (1974) found the exact opposite. "Slow learners" exhibited the highest levels of PS observed and "fast learners" the least. Although there are probably many variables contributing to these differences, one hypothesis with experimental support is the difference due to strain of animal. Pronounced differences in the character of PS increases in different strains of mice have been reported (Smith et al., 1974) and have recently become even more confusing (Kitahama, Valatx, \& Jouvet, 1975). While the PS suppressing qualities of the drug Alphamethyl DOPA retarded the acquisition of a Y-maze shock avoidance task in one strain of mice (C57BR), it seemed to enhance acquisition in the other strain (C57BL/6) (Kitahama et al., 1975).

\section{REFERENCES}

Dement, W., Henry, P., Cohen, H., \& Ferguson, J. Studies on the effect of REM deprivation in humans and in animals. In S. S. Kety, E. V. Evarts, \& H. L. Williams (Eds.), Sleep and altered states of consciousness. Proceedings of the Association for Research in Nervous and Mental Disease. Baltimore: Williams and Wilkins, 1967. Pp. 456-468.

Fishbein, W. Interference with conversion of memory from short term to long term storage by partial sleep deprivation. Communications in Behavioral Biology, 1970, 5, 171-175.

Fishbein, W. Disruptive effects of rapid eye movement sleep deprivation on long term memory. Physiology and Behavior, 1971, 6, 279-282.

Fishbein, W., Kastaniotis, C., \& Chattman, D. Paradoxical sleep: Prolonged augmentation following learning. Brain Research, 1974, 79, 61-75.

Hennevin, E., Leconte, P., \& Bloch, V. Augmentation de sommeil paradoxal provoquée par l'acquisition, l'extinction et la réacquisition d'un apprentissage à reinforcement positif. Brain Research, 1974, 70, 43-54.

Horne, J. A., \& Walmsley, B. Daytime visual load and the effects upon human sleep. Psychophysiology, 1976, 13, 115-120.

Kitahama, K., Valatx, J.-L., \& Jouvet, M. Action différente de l' $\alpha$-methyl-DOPA sur le sommeil et l'acquisition d'un labyrinthe chez deux souches consanguines de souris. Comptes rendus hebdeomadaires des Séances. Academie des Sciences, Paris, 1975, 280, 471-474.

Leconte, P., \& Bloch, V. Deficit de la retention d'un conditionnement aprés privation de sommeil paradoxal chez le rat. Comptes rendus hebdomadaires des Séances. Academie des Sciences, Paris, 1970, 271, 226-229.

Leconte, P., \& Hennevin, E. Augmentation de la durée de sommeil paradoxal consecutif à un apprentissage chez le rat. Comptes rendus hebdomadaires des Seances. Academie des Sciences, Paris, 1971, 271, 86-88.

Leconte, P., \& Hennevin, E. Caracteristiques temporelles de l'augmentation de sommeil paradoxal consécutif à l'apprentissage chez le rat. Physiology and Behavior, 1973, 11, 677-686.

Leconte, P., Hennevin, E., \& Bloch, V. Analyse des effets d'un apprentissage et de son niveau d'acquisition sur le sommeil paradoxal consecutif. Brain Research, 1973, 49. 367-369.

Leconte, P., Hennevin, E., \& Bloch, V. Duration of paradoxical sleep necessary for the acquisition of conditioned avoidance in the rat. Physiology and Behavior, 1974, 13, 675-681.

LUCERo, M. A. Lengthening of REM sleep duration consecutive to learning in the rat. Brain Research, 1970, 20, 319-322.

Pearlman, C. A. Latent learning impaired by REM sleep deprivation. Psychonomic Science, 1971, 25, 135-136.

Pearlman, C. REM sleep deprivation impairs latent extinction in rats. Physiology and Behavior, 1973, 11, 233-237.

Pearlman, C., \& Becker, M. Brief posttrial REM sleep deprivation impairs discrimination learning in rats. Physiology and Behavior, 1973, 1, 373-376.

Pearlman, C., \& Becker, M. REM sleep deprivation impairs bar-press acquisition in rats. Physiology and Behavior, 1974. 13, 813-817. (a)

Pearlman, C. A., \& Becker, M. REM sleep deprivation impairs serial reversal and probability maximizing in rats. Physiological Psychology, 1974, 2, 509-512. (b)

Pearlman, C. A., \& Greenburg, R. Posttrial ReM sleep: A critical period for consolidation of shuttlebox avoidance. Animal Learning and Behavior, 1973, 1, 49-51.

Smith, C. T., Kitahama, K., Valatx, J.-L., \& Jouvet, M. Sommeil paradoxal et apprentissage chez deux souches 
consanguines de souris. Comptes rendus hebdomadaires des Seances. Academie des Sciences, Paris, 1972, 275, 1283-1286.

Smith, C. T., Kitahama, K., Valatx, J.-L., \& Jouvet, M. Increased paradoxical sleep in mice during acquisition of a shock avoidance task. Brain Research, 1974, 77, 221-230.

Smith, C. T., \& Lowe, D. G. Insignificant changes in paradoxical sleep during acquisition of a discrimination avoidance task. Sleep Research, 1975, 4, 153.

Smith, C. T., \& Miskiman, D. E. Increases in paradoxical sleep as a result of amygdaloid stimulation. Physiology and Behavior, $1975,15,17-19$.

Stern, W. C. Acquisition impairments following rapid eye movement sleep deprivation in rats. Physiology and Behavior, 1971, 7, 345-352.

WINER, B. J. Statistical principles in experimental design. New York: McGraw-Hill, 1971.

(Received for publication March 18, 1977; accepted July 7, 1977.) 